\title{
ANALYSIS OF ENDOPLASMIC RETICULUM OF TOBACCO CELLS USING CONFOCAL MICROSCOPY
}

\author{
BARBORA RADOCHOVÁ ${ }^{1}$, JiŘí JANÁČEK ${ }^{1 凶}$, KATEŘINA SCHWARZEROVÁ ${ }^{2}$, ERNA DEMJÉNOVÁ ${ }^{3}$, \\ ZOLTÁN TOMORI $^{3}$, PETR KAREN ${ }^{1}$ AND LuCIE KUBÍNOVÁ ${ }^{1}$ \\ ${ }^{1}$ Department of Biomathematics, Institute of Physiology, Academy of Sciences of the Czech Republic, \\ Vídeňská 1083, Prague 4, CZ-14220, Czech Republic; ${ }^{2}$ Department of Plant Physiology, Faculty of Natural \\ Sciences, Charles University in Prague, Viničná 5, Prague 2, Czech Republic; ${ }^{3}$ Institute of Experimental \\ Physics, Slovak Academy of Sciences, Watsonova 47, 04353 Košice, Slovak Republic \\ e-mail: barborar@biomed.cas.cz, janacek@biomed.cas.cz, karen@biomed.cas.cz, kubinova@biomed.cas.cz, \\ schwarze@natur.cuni.cz,demjen@saske.sk, tomori@saske.sk \\ (Accepted October 13, 2005)
}

\begin{abstract}
Image analysis techniques for preprocessing, segmentation and estimation of geometrical characteristics of fiber-like structures from 2-D or 3-D images captured by a confocal microscope are presented. Methods are demonstrated on fiber-like biological structure: endoplasmic reticulum (ER) of tobacco cells. In the presented analysis of 2-D images of ER before and after the treatment of latrunculin B, ER and ER tubules were segmented and the area density of ER as well as the length density of ER tubules in the cell cortical layer were estimated by automatic image analysis algorithms. Images of 3-D arrangement of ER were reconstructed and rendered by various visualization techniques.
\end{abstract}

Keywords: capillaries, confocal microscopy, endoplasmic reticulum, fibers, image analysis, length density.

\section{INTRODUCTION}

Recent development of imaging methods enables acquisition of images of increasing variety of objects at different scales of magnification. In many studies in biological and materials research it is desirable to evaluate structures that can be regarded as sets of onedimensional (1-D) curves in three-dimensional (3-D) space, i.e., fiber-like structures, such as cytoskeleton, DNA, neuronal extensions, capillaries, root hairs, textile or composite materials. Among contemporary image acquisition techniques, confocal microscopy is especially useful as it enables to obtain perfectly registered stacks of digital images of thin serial optical sections within thick specimens (Pawley, 1995), i.e., 3-D image data, in a large variety of biological samples, including living specimens.

In objects containing or consisting of fiber-like structures, different geometrical characteristics can be of interest, e.g. length or length density, number of branchings, degree of anisotropy. The length of fiberlike structures can be estimated by interactive stereological methods applied to 2-D sections (Mattfeldt et al., 1990) or 3-D subsamples (Gokhale, 1990; Larsen et al., 1998; Mouton et al., 2002), such as confocal stacks of the objects under study. Automatic image analysis techniques are applicable to the estimation of fiber length and other characteristics (Meyer, 1992; Kubínová et al., 2002), provided the relevant 2-D or 3-D grayscale digital images can be segmented, i.e., converted into a binary, black-and-white image with white foreground pixels/voxels belonging to the objects of interest. In such case it is possible not only to evaluate a large number of samples in a short time but also to obtain 3-D reconstructions of structures under study.

In the present study we introduce image analysis techniques for preprocessing, segmentation and estimation of geometrical characteristics of fiber-like structures from 2-D or 3-D images captured by a confocal microscope using our special custom-made modules developed in the IRIS Explorer (NAG, UK) visualization environment and our plug-in modules running in Ellipse (ViDiTo, Slovakia) environment. Methods are demonstrated on the example of endoplasmic reticulum of tobacco cells.

The endoplasmic reticulum (ER) is a membranous structure that fulfils many important functions within a eukaryotic cell. It forms a 3-D lace-like net consisting of continuous tubules and flat sheets whose motility 


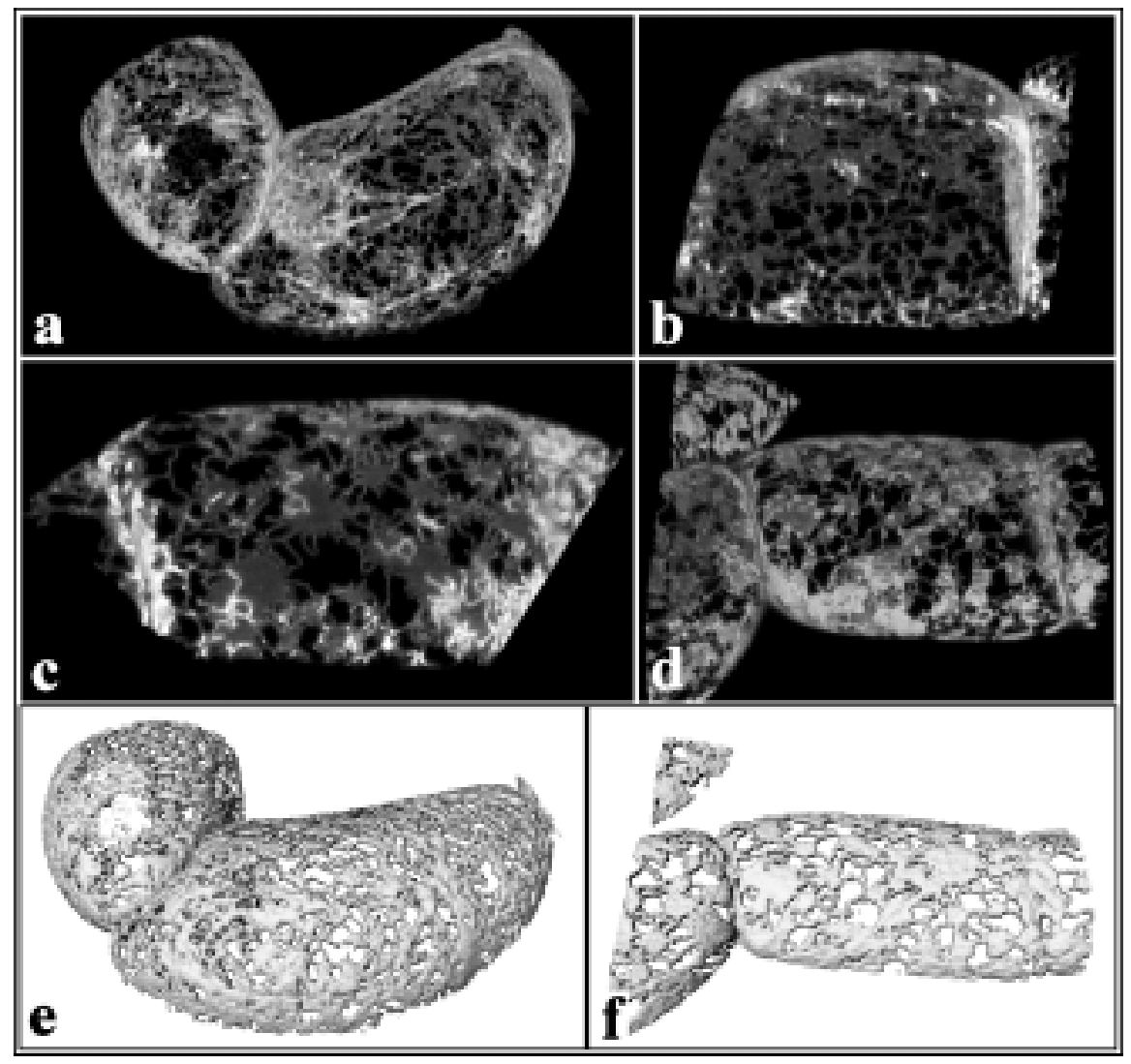

Fig. 1. 3-D visualization of endoplasmic reticulum of tobacco cells. Volume (a-d) and surface $(e, f)$ rendering of control $(a, b, e)$ cells and cells exposed to latrunculin $B(c, d, f)$. The original image data are represented by stacks of confocal images: 68 serial images with voxel size of $156 \times 156 \times 611 \mathrm{~nm}^{3}(a, e), 72$ serial images with voxel size of $70 \times 70 \times 122 \mathrm{~nm}^{3}$ (b), 63 serial images with voxel size of $70 \times 70 \times 122 \mathrm{~nm}^{3}(\mathrm{c})$, and 55 serial images with voxel size of $135 \times 135 \times 611 \mathrm{~nm}^{3}(d, f)$.

can be observed in a living cell as a flow of tubular ER structures in cytoplasmic strands or changes in tubular structures and lamellar sheets (Fig. 1). These changes comprise movement of tubules as well as their growth and shrinkage. It was shown that motility of tubules of ER net is actomyosin-dependent and that drugs interfering with actin cytoskeleton interfere also with motility of ER structures (Liebe and Menzel, 1995). A number of different functions carried out by ER within a cell is associated with different types of ER membranes, the proportion of which can change, e.g., in relation to the developmental phase or stress conditions. It would be interesting to find out if actin cytoskeleton plays an important role not only in motility, but also in structural reorganization of different types of ER cisternae in plant cells. We have used latrunculin B, an actin-depolymerising drug, to destabilise actin cytoskeleton in tobacco cells BY-2 and to observe changes in structure of cortical ER.

\section{MATERIALS}

Latrunculin B (LatB, Sigma Aldrich) was diluted in DMSO in a stock solution $2.53 \mathrm{mM}$. LatB from the stock solution was added directly into the culture medium of exponentially growing, three-day-old tobacco cells (line BY-2), expressing pBIN $m-g f p 5$ ER plant binary vector, to a final concentration of $500 \mathrm{nM}$. For analysis of differences between control cells and cells exposed to LatB, images of thin optical sections of the cortical layer in individual cells, sampled at random, were captured by a Leica SP2 AOBS laser scanning confocal microscope using $63 \times$ water immersion objective $(\mathrm{NA}=1.2)$ with $15 \times$ zoom and 1-photon excitation of GFP (excitation wavelength $488 \mathrm{~nm}$ ) (Figs. 2a,3a). For 3-D visualization of ER arrangement, series of optical images were acquired (Fig. 1). 
In order to test the developed image analysis methods, 13 control cells and 10 cells, treated with LatB at different time of cultivation ranging from 5 to $50 \mathrm{~min}$, were analyzed.

\section{METHODS}

For the visualization of the 3D images (Fig. 1) the volume rendering was employed using a custom made module of the Ellipse software (VIDITO, Slovakia) and VP1000 (TerraRecon, USA) visualization card.

For 2-D analysis of endoplasmic reticulum the 2D grayscale images were resampled to size $256 \times 256$ with pixel size of $62 \mathrm{~nm} \times 62 \mathrm{~nm}$. Regions of interest were selected interactively, according to visual sharpness of the texture (Figs. 2a,3a). Images were processed by a median filter of size $3 \times 3$ to remove noise. Endoplasmic reticulum (consisting of tubules and lamellar sheets) was detected by thresholding at local minimum of histogram of image intensities (Figs. 2b,3b). The area density of ER in the cell cortical layer, $A_{A}(E R)$, was estimated by dividing the sum of the areas of pixels lying inside ER by the area of the region of interest. Further, the length density of ER tubules in the cell cortical layer, $\mathrm{L}_{\mathrm{A}}(\mathrm{ER})$, was estimated. For this purpose, ER tubules were enhanced using morphological grayscale hit or miss opening (Soille, 2003) with two sizes of structuring element, i.e., with segment of length $F(=6,8)$ pixels as a foreground structuring element and two parallel segments $\mathrm{B}(=3,5$ pixels $)$ long and $\mathrm{D}(=4,5)$ pixels apart from the foreground element (Fig. 4), repeating the procedure for 24 orientations of the element (from $0^{\circ}$ to $\left.180^{\circ}\right)$, multiplying by a constant $\mathrm{C}(=1,0.8)$ and taking maximum of corresponding values from the $2 \times 24=48$ images (Fig. 2c, 3c).

Binary images of the tubules were then obtained by thresholding. To obtain a geometrical representation of the tubules, the skeleton of the binary images was constructed from boundary segments of cells of Voronoi mosaic of boundary pixels (Figs. 2d,3d), pruning the skeleton branches by the distance between the generating point along the boundary after Ogniewicz and Ilg (1992) and subsequently pruning the short end segments. The mosaic was calculated by Euclidean distance transform after Breu et al. (1995). The skeleton was regularized by an active contours (snake) algorithm. $\mathrm{L}_{\mathrm{A}}(\mathrm{ER})$ was estimated by dividing the sum of the segment lengths by the area of the region of interest. The variants were statistically compared by two-sample Student t-test.
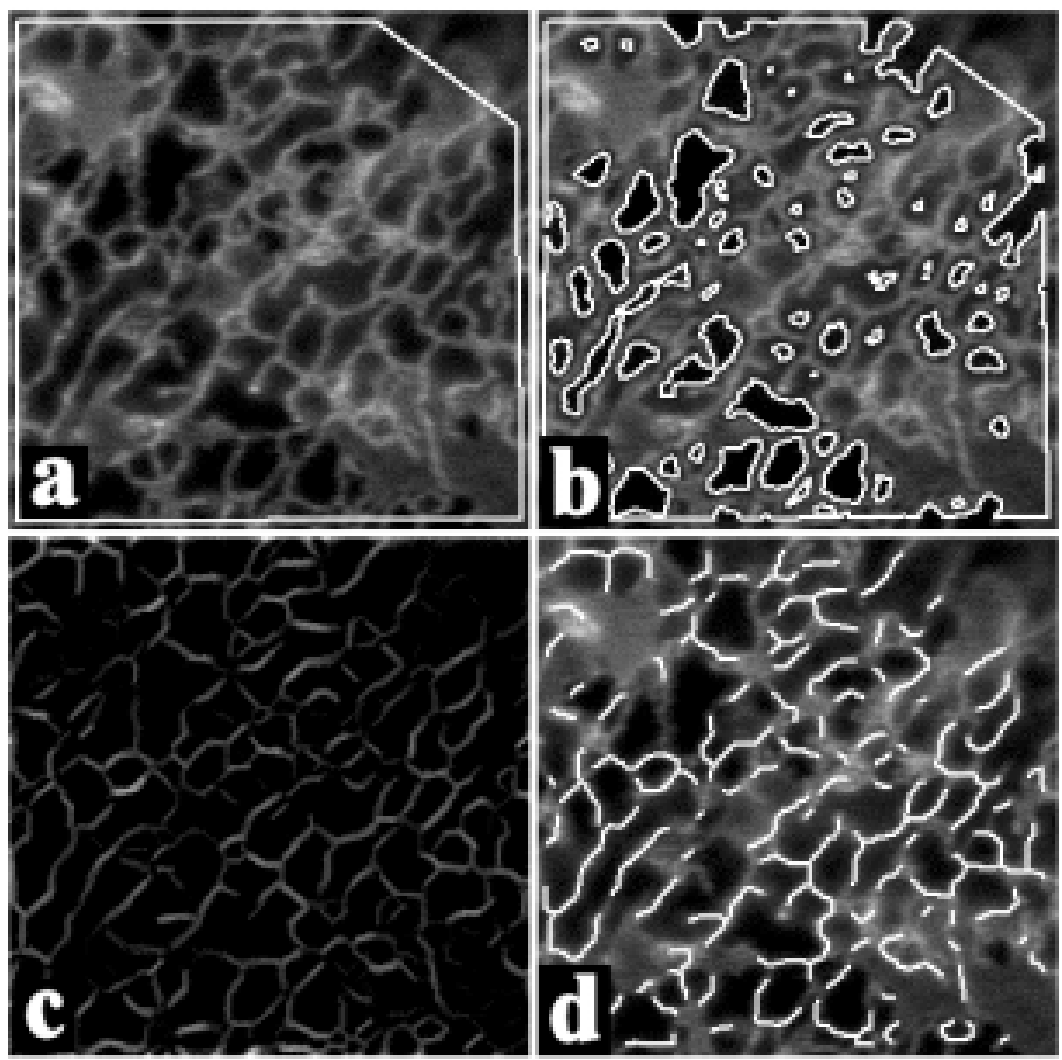

Fig. 2. Confocal images of endoplasmic reticulum in a control tobacco cell. (a) Original image with the detected region of interest; (b) image with boundaries of ER; (c) image with enhanced tubules; (d) image with skeleton smoothed by snake transform. The dimensions of the original images are $15.9 \mu \mathrm{m} \times 15.9 \mu \mathrm{m}$. 

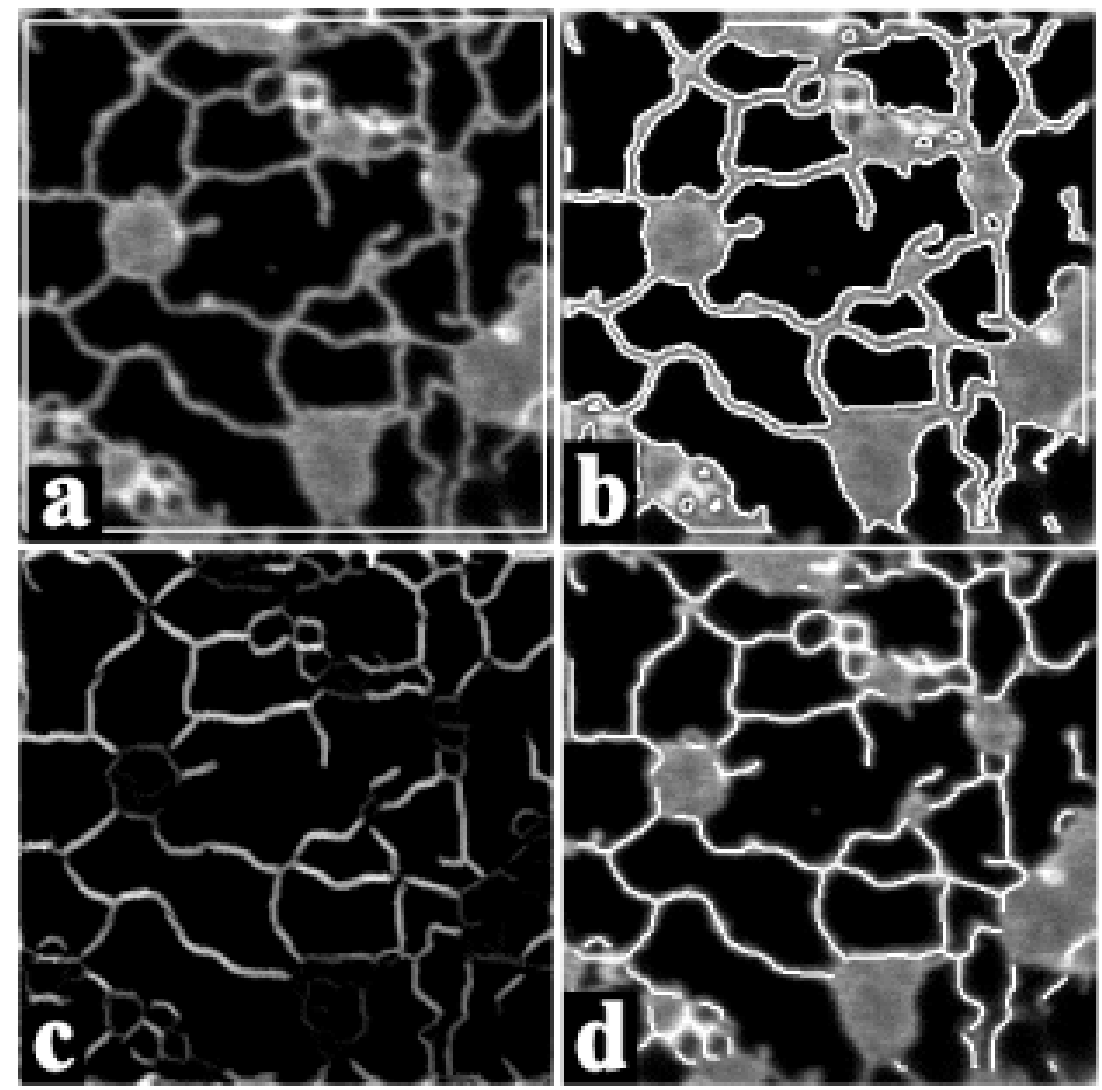

Fig. 3. Confocal images of endoplasmic reticulum in a cell treated by latrunculin B for 20 min (a) Original image with the detected region of interest; (b) image with boundaries of ER; (c) image with enhanced tubules; (d) image with skeleton smoothed by snake transform. The dimensions of the original images are $15.9 \mu \mathrm{m} \times 15.9 \mu \mathrm{m}$.

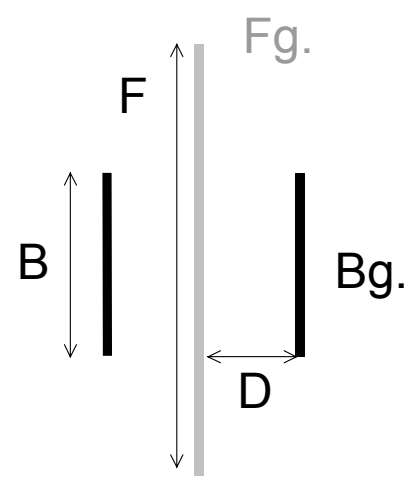

Fig. 4. Structuring element for hit-or-miss opening with size parameters $F, B, D$.

\section{RESULTS}

Based on the analysis of 13 control cells and 10 cells exposed to LatB, we found a significant difference both in the area density of ER in the cell cortical layer, $A_{A}(E R)$, and the length density of ER tubules in the cell cortical layer, $\mathrm{L}_{\mathrm{A}}(\mathrm{ER})$ in cells exposed to latrunculin $\mathrm{B}$ (mean $\mathrm{A}_{\mathrm{A}}(\mathrm{ER})=0.617, \mathrm{SD}=0.203$; mean $\left.\mathrm{L}_{\mathrm{A}}(\mathrm{ER})=0.76 \mu \mathrm{m}^{-1}, \mathrm{SD}=0.16 \mu \mathrm{m}^{-1}\right)$ when compared with control cells (mean $\mathrm{A}_{\mathrm{A}}(\mathrm{ER})=0.776$, $\mathrm{SD}=0.073$; mean $\mathrm{L}_{\mathrm{A}}(\mathrm{ER})=1.02 \mu \mathrm{m}^{-1}, \mathrm{SD}=0.15$ $\left.\mu \mathrm{m}^{-1}\right)$.

\section{DISCUSSION}

The proposed methods enabled to estimate geometrical characteristics of fiber-like structures of interest in biological studies. Results for endoplasmic reticulum of tobacco cells showed that the area density of ER in the cell cortical layer is lower in latrunculin B-treated cells. This indicates that without the support of actin cytoskeleton, which was disrupted by latB treatment, tubules and sheets of ER fused into bigger compartments, accompanied by the reduction of ER total area. Further, the length density of ER tubules in the cell cortical layer was lower in latBtreated cells. This result can be explained by the 
decreased complexity of ER net in the absence of actin cytoskeleton. The presented pilot analysis suggests that actin cytoskeleton plays a role in the organisation of structure of cortical ER.

The presented methods can be applied to other fiber-like structures, though some adaptation to specific situation would be needed. In this study we have focused on measurements of the most important characteristic of the fiber-like structures, i.e., length or length density. To describe finer properties of arrangement of fibers it will be necessary to measure their directional distribution to characterize anisotropy or correlation function to describe spatial regularity or clustering of the fibers.

\section{ACKNOWLEDGEMENTS}

The study was supported by the Academy of Sciences of the Czech Republic (Grant KJB6011309).

\section{REFERENCES}

Breu H, Gil J, Kirkpatrick D, Werman M (1995). Linear Time Euclidean Distance Transform Algorithms. IEEE TPAMI: IEEE Trans Pattern Anal Machine Intelligence 17:529-33.

Gokhale AM (1990). Unbiased estimation of curve length in 3D using vertical slices. J Microsc 159:133-41.
Kubínová L, Janáček J, Krekule I (2002). Stereological methods for estimating geometrical parameters of microscopical structure studied by three-dimensional microscopical techniques. In: Diaspro A, ed. Confocal and Two-photon Microscopy. New York: Wiley-Liss, 299-332.

Larsen JO, Gundersen HJG, Nielsen J (1998). Global spatial sampling with isotropic virtual planes: estimators of length density and total length in thick, arbitrarily orientated sections. J Microsc 191:238-48.

Liebe S, Menzel D (1995). Actomyosin-based motility of endoplasmic reticulum and chloroplasts in Vallisneria mesophyll cells. Biol Cell 85:207-22.

Mattfeldt T, Mall G, Gharehbaghi H, Moller P (1990). Estimation of surface area and length with the orientator. J Microsc 159:301-17.

Meyer F (1992). Mathematical morphology: from two dimensions to three dimensions. J Microsc 165:5-28.

Mouton PR, Gokhale AM, Ward NL, West MJ (2002). Stereological length estimation using spherical probes. J Microsc 206:54-64.

Ogniewicz R, Ilg M (1992). Voronoi Skeletons: Theory and Applications. Proc. CVPR'92, 1992; Champaign, Illinois, 63-9.

Pawley JB (1995). Handbook of Biological Confocal Microscopy. Second Edition. New York: Plenum Press.

Soille P (2003). Morphological Image Analysis, Principles and Applications, $2^{\text {nd }}$ edition. Springer. 